\title{
APPARATUS FOR AUTOMATIC CHEMICAL ETCHING OF METALLOGRAPHIC SAMPLES
}

\author{
1'Ondřej AMBROŽ, ${ }^{2} J a n$ ČERMÁK, ${ }^{3}$ Šárka MIKMEKOVÁ \\ Institute of Scientific Instruments of the CAS, Brno, Czech Republic, EU, \\ 'ondrej@isibrno.cz, ${ }^{2}$ cermak@isibrno.cz, ${ }^{3}$ sarka@isibrno.cz
}

https://doi.org/10.37904/metal.2021.4146

\begin{abstract}
The microstructure of steels after mechanical polishing is revealed only by the application of a suitable etchant. To achieve adequate optical or electron microscope images, the specimen surface must be free of any artifacts. Chemical etching can be defined as a controlled corrosion process. The metal of the investigated material passes as cations into the etchant solution during the chemical etching reaction. Chemical etching is usually performed manually either by immersing the sample in the etchant with simultaneous stirring or by swabbing with a lint-free cloth soaked in the etchant. It is also extremely important to debug the process of removing the sample from the bath and subsequent cleaning. It is recommended to wash the sample after removal from the etchant with water (distilled or demineralized) or alcohol (ethanol, methanol, or isopropyl alcohol) and dry it properly (depending on the etchant and the etched material). The main problem with these processes is the human factor, which significantly contributes to the already limited repeatability. All operation steps must be performed by properly trained personnel in the field of occupational safety because hazardous substances are handled. A high manual dexterity is also needed. Training a new employee is a long-term process. Moreover, keeping the exact etching time can be a challenge and one second can decide success. These problems become more serious in the case of using surface sensitive analytical method, such as a low energy scanning electron microscopy, due to the high spatial resolution and extreme surface sensitivity. We have developed an apparatus for automatic etching of metallographic samples of purpose to overcome all above-mentioned difficulties. The apparatus and results of the first experiments will be presented.
\end{abstract}

Keywords: Metallography, chemical etching, automation, SEM, ultrasonic bath

\section{INTRODUCTION}

Many etchants were developed on the basis of empirical methods only with knowledge of the corrosion behaviour of the metals. Established etchants usually consist of three components: a corrosive agent (such as hydrochloric, sulfuric, acetic, or phosphoric acid), a modifier (such as ethanol or glycerin) that reduces ionization, and an oxidizing agent (such as hydrogen peroxide, $\mathrm{Fe}^{3+}$ or $\mathrm{Cu}^{2+}$ ). One component can perform two functions as nitric acid in Nital. Oxygen dissolved in tap water increases the etching rate, so it is always better to use distilled water to prepare etchants. The success of etching depends on many factors, such as the order in which the substances are mixed, their purity and freshness. If the polished surface is etched upwards, the surface can be observed, which allows the process to be stopped at the right time. This procedure is preferred for conventional manual etching, especially in the case of the first etching of the unknown effect of an etchant. The etching with a polished surface downward is used to minimize settling of reaction products on the surface. The sample or the solution is stirred for the same reason. The etching is usually performed by immersion in a solution at room temperature. Adhering to the etching time is important to obtain a contrasting image that allows distinguish fine details, but it is difficult to control a process that takes a few seconds [1]. It is usually not recommended to etch several times, especially for etchants forming stable layers. If the results are 
unsatisfactory, the samples must be repolished and the process repeated. The process of withdrawing the sample from the etchant, as well as washing and drying, must be carefully optimized. There must be no local drying of the sample surface between these processes [2]. It is recommended to clean the sample in several stages with water or a suitable alcohol after etching (depending on the material and the etchant). It is advisable to include washing the sample in an ultrasonic bath in the process. The sample is placed in a beaker of cleaning solution in this case. And this beaker is placed in an ultrasonic bath. The use of a beaker guarantees greater flexibility. Changing the cleaning solution will be easier and the consumption of the cleaning solution will be lower. The cleaning of samples in an ultrasonic bath should not exceed 30 seconds for very soft materials [3]. The etching of heterogeneous alloys, which also includes the multiphase TRIP steel investigated in this work, emphasizes the surface relief. The individual phases differ in their electrode potential and thus each phase also has a different corrosion resistance. The tendency of metals to dissolve in solution depends on their position in the electrochemical series. This also applies to chemically differently composed phases, which also create potential differences [4]. Different phases dissolve at different rates during the etching process. There may be different roughening of the surfaces of the individual phases. Selective etching is more difficult to perform successfully because a preferential chemical reaction is required and this problem multiplies with multiphase steels. The etching time must be strictly controlled to avoid the overetching. Multiphase steels generally have shorter etching times than single phase steels [5]. When observing etched specimen of multiphase steels in a light microscope, the cathodic phase emerges from the relief and has a light shade, while the anodic phase is embedded in the depth of the sample and may have a rough surface depending on the composition of the etchant and the tested sample. The increased roughness of the anodic phase is manifested by a darker shade in the light microscope. One of the rules says that the higher the magnification planned to observe the structure, the shorter the etching time should be and vice versa [1]. The chemical etching remains an empirical method. This results from a number of parameters entering into this process. Each specific material and sample needs to be approached individually. It is useful to know the history of the tested material, which can help us in the preparation itself. It is necessary to focus on the process parameters that have the greatest impact and optimize them. The vast majority of these techniques have been developed for light microscopy. The scanning electron microscopy requires even more careful preparation. Partial automation could solve at least some of these problems.

\section{EXPERIMENTAL TECHNIQUES}

The TRIP steel $0.2 \mathrm{C}-1.5 \mathrm{Si}-2 \mathrm{Mn}$ sheet of $1.6 \mathrm{~mm}$ thick was cut into $9 \mathrm{~mm}$ square samples. The samples were mechanically ground and polished to a final step with diamond paste of $0.25 \mu \mathrm{m}$ particles and cooled with isopropyl alcohol. The etching, cleaning and drying were performed automatically on the apparatus described below.

An apparatus for automatic etching of metallographic specimens (Figure 1) consists of an aluminium frame construction (1) for ensuring accurate mutual position of an ultrasonic bath (3) with a beaker (5) ceramic glass holder (4) and a 5-axis robotic arm (6) with a detachable cross tweezers (8) as replacement of a robot end effector. Tweezers (8) are mounted via the 3D printed ASA plastic part (9), which is connected to the robotic arm's flange. A specimen (7) is gripped between a tweezers' jaws.

To the aluminium frame construction are attached PET-G panels (2) for chemicals splash prevention. Access to a PET-G box is provided by a front door that is locked in close position by magnets. In the PET-G panels there are laser-cutted holes to attach a rear connector panel and a power socket. A robotic arm control unit and a holder for a heat gun are attached to the PET-G panels as well. Specimen is dried by the heat gun (11) in this position. Fumes from etching process are extracted from the box by a fume hood. Fume hood is connected by PTFE flexible ducting hose, which is attached to the box by a flange (10). 


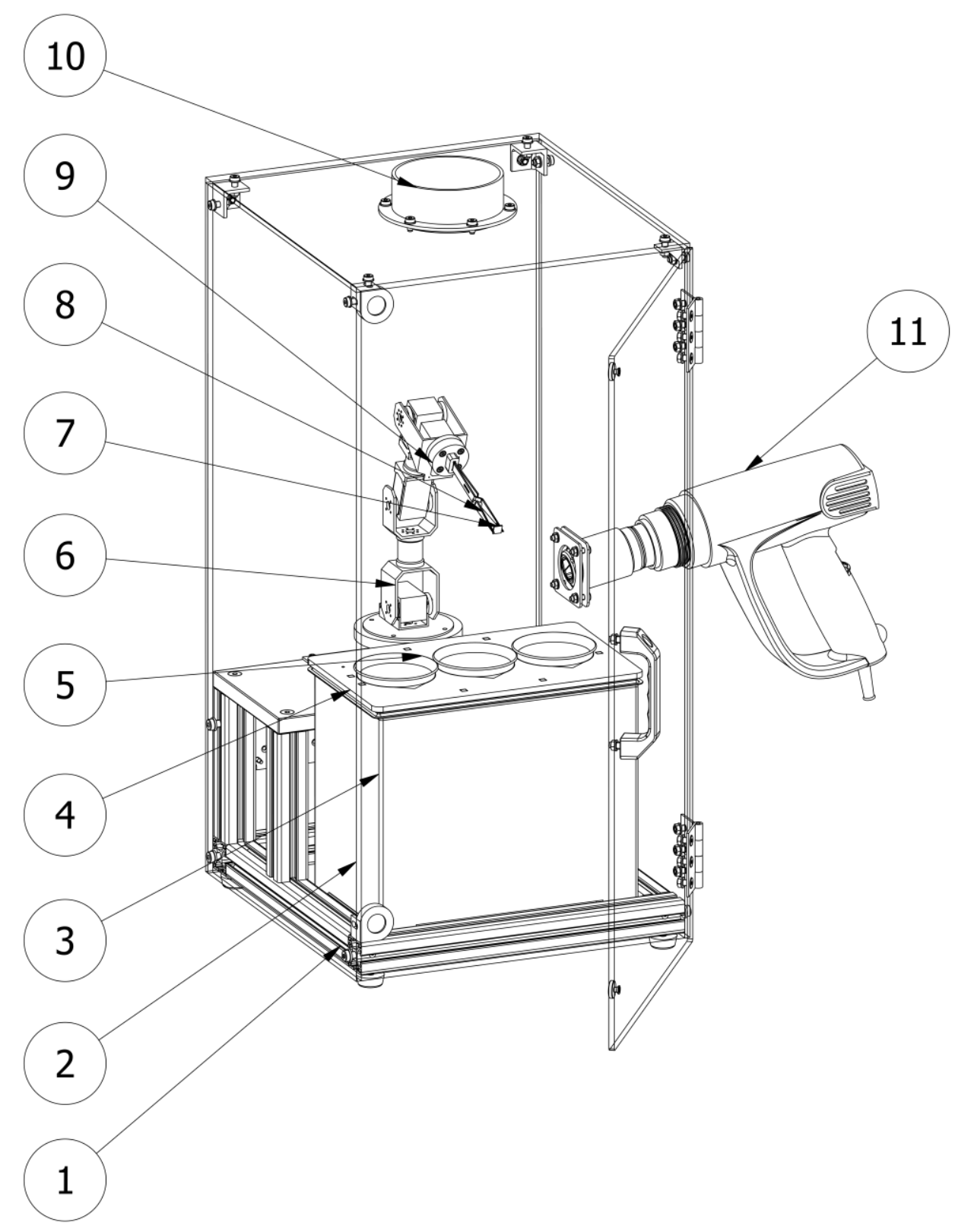

Figure 1 Etching apparatus

The five-axis robotic arm (5DoF) with serial kinematics consists of five rotary axes. The movement is provided by intelligent programmable DC actuators using the serial bus protocol. The actuators are controlled by a programmable servo controller based on a single-chip ARM STM32 microcomputer. The arm is programmed by writing an angle of the rotation of the individual axes at a specific point, the angular range of the axes in the servo mode is $240^{\circ}$. The resulting programmed movement is determined by the composition of the partial programmed movements between the individual points. The arm moves between the points with a PTP (PointTo-Point) movement. The speed of movement between points is given by the required travel time in milliseconds.

After a mechanical polishing, the specimen was gripped in the tweezers. Then the tweezers were mounted on the robotic arm. In a next step crew member played the ultrasonic bath, closed the door of the safety box, switched on the heat gun and via a laptop ran the robotic arm's program. After an automated process was finished, crew member switched off the heat gun, opened the safety box door, stop the ultrasonic bath and then he unmounted the tweezers with the specimen. 
The automated process is driven by a user code and consists of twelve main steps. A robotic arm's movement sequence is displayed in Figure 2:

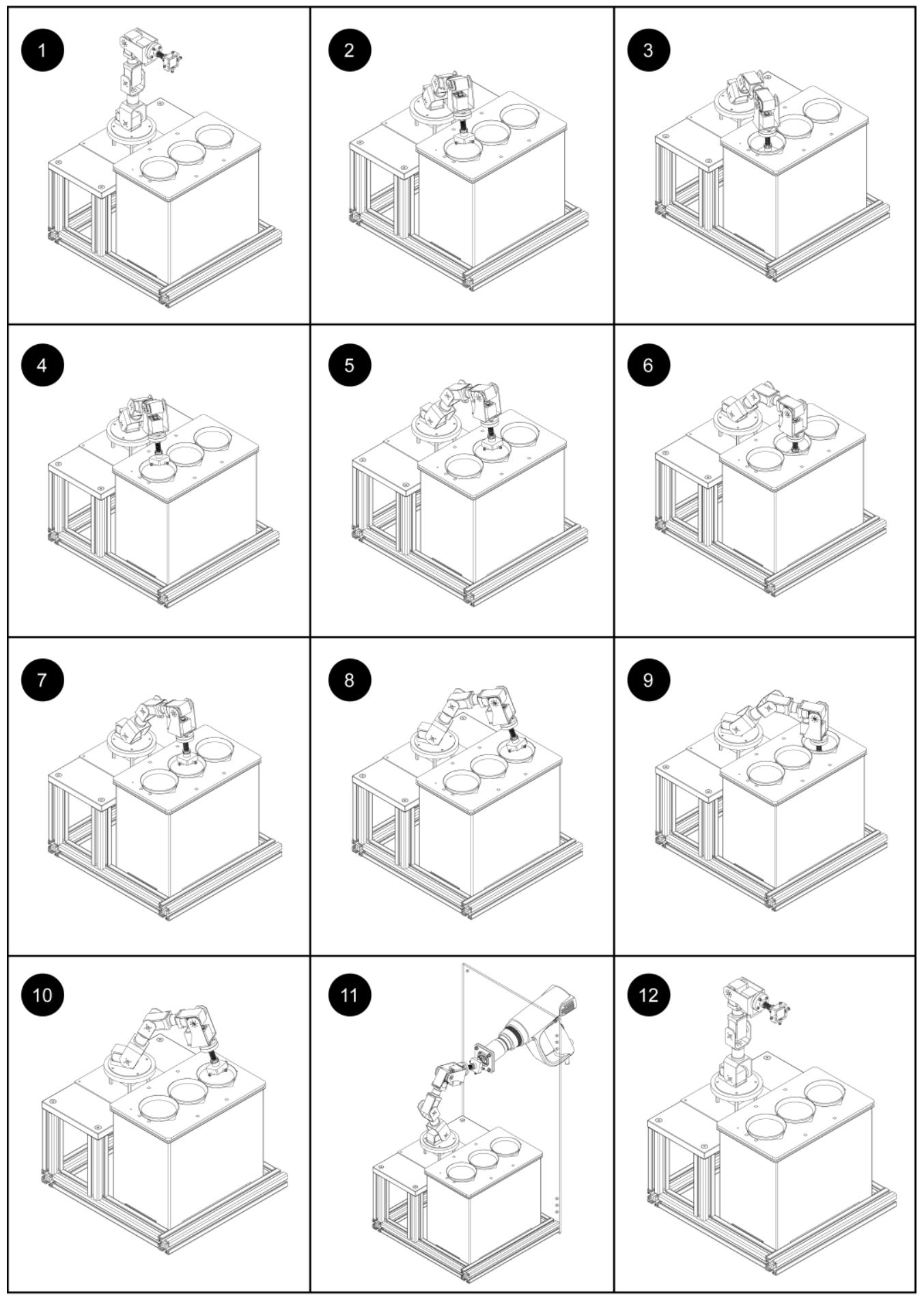

Figure 2 Robotic arm movement sequence

1) In this position crew member mount the tweezers with specimen on the arm.

2) The robotic arm moves from home position to above of the beaker with etchant.

3) The robotic arm immerses tweezers with specimen to beaker with etchant for specified time. 
4) The robotic arm is moved above the beaker again.

5) The position is changed to an upward of the second beaker with a cleaning liquid.

6) The robotic arm is immersed to the cleaning liquid for specified time.

7) The robotic arm emerges.

8) The robotic arm moves above the third beaker with another cleaning liquid.

9) The robotic arm immerses to the cleaning liquid for specified time.

10) The robotic arm rises.

11) The robotic arm moves in a front of the heat gun nozzle for specified time.

12) The robotic arm returns to the initial position.

During the immersion arm executes rapid side to side movement. Time of the immersing movement (step 3 , 6 and 9) was $500 \mathrm{~ms}$. Time of the emerging movement was $200 \mathrm{~ms}$. Time of the step 5 and 8 is $200 \mathrm{~ms}$ long. Time of immersion in the etchant varies according to an experiment setting. Time of immersion in the cleaning liquid is $1000 \mathrm{~ms}$ in the first cleaning beaker or $3000 \mathrm{~ms}$ in the second cleaning beaker.

The most common etchant for Fe alloys Nital was chosen for a pilot experiment to verify the effect of ultrasound on the etching and cleaning process. We used it in the $4 \%$ version. The etching time of $5 \mathrm{~s}$ was chosen according to previous experiments. In the first case, the ultrasound was turned off during the whole process. In the second case, the ultrasound was turned on during the whole process, and in the latter case, it was turned on only for the cleaning process. The ultrasound was set to $80 \mathrm{kHz}$ and pulse mode. Methanol was chosen as the cleaning liquid. The heat gun was set to $100^{\circ} \mathrm{C}$ during drying.

The specimen surfaces were observed in an UHR SEM Magellan $400 \mathrm{~L}$ (Thermofisher Scientific) immediately after the preparation. The microscope was operated at $1 \mathrm{keV}$ landing energy of the primary electrons. It secures an extremely high surface sensitivity due to short penetration depth of the primary electron beam. The micrographs created by the secondary and back-scattered electrons were collected simultaneously by a truethe-lens detector (TLD) and a concentric backscatter electron detector (CBS) situated bellow the pole piece. A cathode lens system, i.e. negative biased specimen, secured high detection efficiency at low landing energies [6].

\section{RESULTS AND DISCUSSION}

The SEM micrographs are shown in Figure 3. The immersion time in the etchant $5 \mathrm{~s}$ was sufficient to remove the deformed layer after mechanical preparation and the secondary phases (i.e. martensite and retained austenite) can be distinguished from the ferritic matrix. All samples are without disturbing contamination thanks to the optimal setting of apparatus movements. This is also achieved by using tweezers as a sample holder, where the liquid does not stick in the folds and crevices. The TLD and the CBS micrographs taken at an impact energy of $1 \mathrm{keV}$ show that the use of ultrasound in the etching process seems inappropriate (c). Chemical attack by the etchant in conjunction with ultrasonic cavitation results in the increase of dissolution rate of the matrix, inhomogeneous etching and the formation of microporosity. Without the use of ultrasound (a), these problems do not occur and we are closest to the conditions of classical manual etching, however, with the advantage of repeatability of movements and a precisely determined time in the order of fractions of a second. The use of ultrasound only for cleaning after the etching process (b) seems to be promising. The surface of the matrix and secondary phases is clean without disturbing artifacts. In BSE images acquired by the CBS detector, it is only possible to observe artifacts as remains of scratches on the martensitic phase emerging from the matrix. However, these artifacts are caused by previous mechanical preparation and not by etching. 
a) ultrasonic bath OFF

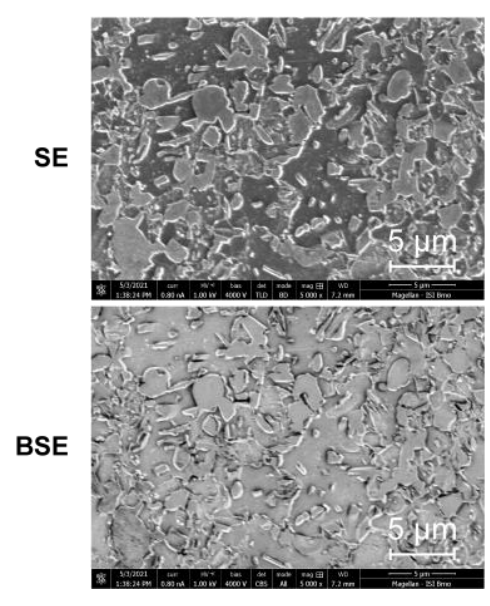

b) ultrasonic bath $\mathrm{ON}$ - cleaning c) ultrasonic bath always $\mathrm{ON}$

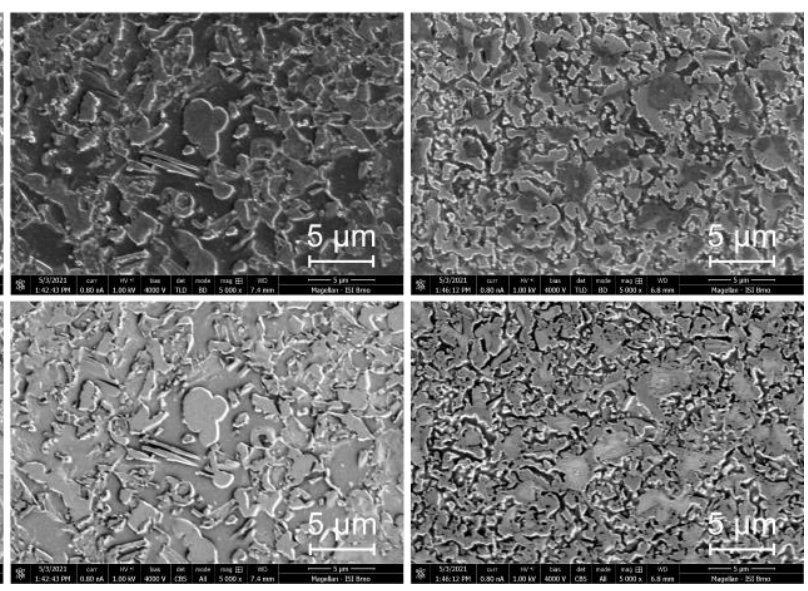

Figure 3 The effect of ultrasound on the etching and cleaning process. The SEM micrographs of the TRIP steel specimen automatically etched in Nital for $5 \mathrm{sec}$ obtained at $1 \mathrm{keV}$ landing energy of the primary beam with the TLD and the CBS detector.

\section{CONCLUSION}

Automated chemical etching was applied on the metallographic samples of TRIP steel. Repeatability was ensured thanks to automated etching, cleaning and drying of the samples. This is difficult to achieve in conventional metallography. Keeping the exact etching time in the order of a fraction of a second is almost impossible by the manual method. The repeatability and validity of the experiments are therefore very low. The apparatus fulfilled its purpose and the suitability of including the ultrasonic bath in the etching process was verified. It is not advisable to use switched on ultrasound during etching. The cavitation occurs and the uniformity of etching decreases. It is advisable to turn on the ultrasound only during the cleaning process. Only one basic etchant and one time were used in this experiment. To fully exploit the potential of the device, further experiments with different etchants and times are planned for the future.

\section{ACKNOWLEDGEMENTS}

\section{The research was supported by Technology Agency of the Czech Republic - National Centre of Competence No. TN01000008.}

\section{REFERENCES}

[1] VANDER VOORT, G.F. Metallography, principles and practice. Materials Park, Ohio: ASM International, 1999.

[2] SKOČOVSKÝ, P., PODRÁBSKÝ T. Farebná metalografia zliatin železa. Žilina: Žilinská univerzita, 2001.

[3] ASM Handbook Volume 9: Metallography and Microstructures. Materials Park, Ohio: ASM International, 2004.

[4] ANGELI, J., FÜREDER, E., PANHOLZER, M., KNEISSL, A.C. Ätztechniken für die Phasencharakterisierung von niedriglegierten Dual-Phasen- und TRIP-Stählen. Practical Metallography. 2006, vol. 43, no. 10, pp. 489-504.

[5] GUVEN, G. Chemical etching technique for porous and fine-grain YBa2Cu307-x superconductors with or without silver addition. Bethlehem, 1992. Lehigh University.

[6] DUFEK, M. Magellan 400L: User Operation Manual. Hillsboro, Oregon: FEI Company, 2011. 